\title{
A novel drug discovery strategy: Mechanistic investigation of an enantiomeric antitumor agent targeting dual p53 and NF-kB pathways
}

\author{
Chunlin Zhuang ${ }^{1,2, *}$, Chunquan Sheng ${ }^{1, *}$, Woo Shik Shin ${ }^{3}$, Yuelin Wu ${ }^{1}$, Jin $\mathbf{L i}^{1}$, \\ Jianzhong Yao ${ }^{1}$, Guoqiang Dong ${ }^{1}$, Wen Zhang ${ }^{2}$, Yuk Yin Sham ${ }^{3}$, Zhenyuan Miao ${ }^{1}$, \\ Wannian Zhang ${ }^{1}$ \\ ${ }^{1}$ Department of Medicinal Chemistry, School of Pharmacy, Second Military Medical University, Shanghai, 200433, People's \\ Republic of China \\ ${ }^{2}$ Research Center for Marine Drugs, School of Pharmacy, Second Military Medical University, Shanghai, 200433, People's \\ Republic of China \\ ${ }^{3}$ Center for Drug Design, Academic Health Center, University of Minnesota, Minneapolis, 55455, Minnesota \\ "These authors contributed equally to this work
}

Correspondence to:

Zhenyuan Miao, e-mail: miaozhenyuan@hotmail.com

Wannian Zhang, e-mail: zhangwnk@hotmail.com

Yuk Yin Sham, e-mail: shamx002@umn.edu

Keywords: p53-MDM2, NF-кB, antitumor activity, dual inhibitors, enantiomer, molecular dynamics, molecular recognition

Received: June 30, 2014

Accepted: September 24, 2014

Published: October 07, 2014

\section{ABSTRACT}

The p53 and nuclear factor KB (NF-KB) pathways play crucial roles in human cancer development. Simultaneous targeting of both pathways is an attractive therapeutic strategy against cancer. In this study, we report an antitumor molecule that bears a pyrrolo[3,4-c]pyrazole scaffold and functions as an enantiomeric inhibitor against both the p53-MDM2 interaction and the NF-kB activation. It is a first-in-class enantiomeric inhibitor with dual efficacy for cancer therapy. Synergistic effect was observed in vitro and in vivo. Docking and molecular dynamics simulation studies further provided insights into the nature of stereoselectivity.

\section{INTRODUCTION}

The p53 tumor suppressor protein is believed to play a crucial role in preventing cancer development by regulating cell cycle and inducing apoptosis [1]. MDM2 protein is a negative regulator of the $\mathrm{p} 53$ pathway and its overexpression in cancer cells may lead to inactivation of p53 cellular function. Disruption of the p53-MDM2 interaction is expected to overcome the oncogenic consequences of MDM2 overproduction and restores p53 function [2]. The design and identification of potent p53-MDM2 inhibitors have been avidly pursued in the past decade and several non-peptide small inhibitors such as nutlins [3], benzodiazepines [4], spirooxindoles [5], and piperidinones [6] have been developed as anticancer agents.

The NF- $\mathrm{kB}$ is another central mediator of cellular response to external stimuli [7] involved in the control of inflammation, apoptosis and cell proliferation in the human body $[8,9]$. Under basal conditions, the NF- $\mathrm{kB}$ complex responsible for regulating the transcription of DNA is suppressed by a family of inhibitors called IкBs in the cytoplasm via protein association. Under stimulated conditions, the IкB inhibitory proteins are phosphorylated by I $\mathrm{KB}$ kinase (IKK) composed of $\alpha, \beta$ and $\gamma$ subunits, resulting in ubiquitination and eventual degradation of the I $\kappa$ Bs. The dissociated NF- $\kappa B$ complex subsequently enters the nucleus where it binds to DNA and activates gene expression. Therefore, inhibition of NF- $\mathrm{kB}$ signalling by preventing the IKK phosphorylation of IKB proteins has potential therapeutic applications to the treatment of cancer and inflammatory diseases [10].

Recent analysis showed many NF- $\kappa \mathrm{B}$ repressors may also function as p53 activators and vice versa $[11,12]$. Regulation of the cross-talk between the p53 and the NF- $\mathrm{kB}$ pathways would offer a unique therapeutic opportunity for targeting [11]. However, the promiscuous interaction of a small molecule with off-target proteins 
frequently causes toxicity [13] and adverse effects $[14,15]$. Conversely, a single drug target can be therapeutically insufficient, particularly in complex neuropsychiatric conditions, cancers and infectious diseases [16]. Designing compounds with a specific multi-target profile is both complex and challenging $[17,18]$. The problem of designing molecules against a multi-target profile involves the parallel optimization of multiple structure-activity relationships within a desired range of physicochemical properties [19].

In our recent study [20], a distinct effort was made to design a p53-MDM2 interaction inhibitors bearing pyrrolidone system through structure-based virtual screening. Subsequently, a novel pyrrolo[3,4-c]pyrazole was rationally designed as a potent inhibitor of the p53MDM2 protein-protein interaction and the NF- $\kappa$ B pathway. A dual inhibitor is preferred over the combined use of two single-target $\mathrm{p} 53-\mathrm{MDM} 2$ and NF- $\mathrm{BB}$ inhibitors because it eliminates the need to optimize individual inhibitor doses for efficacy and the potential complications of drug-drug interactions. In this study, racemic pyrrolo[3,4-c]pyrazole was demonstrated as a first-in-class small molecule being used simultaneously as a p53-MDM2 and NF- $\kappa$ B inhibitor. The p53-MDM2 inhibition was confirmed by cell-free biochemical assay and cell-based Western blot. Meanwhile, the racemic compound was also shown to suppress NF- $\kappa$ B nuclear translocation by inhibiting the I $\mathrm{B}$ kinases of NF- $\kappa \mathrm{B}$ pathway [21].

The central goal of this study is to determine whether the two enantiomers (see Supplementary information for NMR spectra and chiral separation, Figure S1) of the newly designed racemic compound [21] of 5-(3-(1H-imidazol-1-yl)propyl)-4-(4-bromo-phenyl)1-(4-fluorobenzyl)-3-phenyl-4,5-dihydropyrrolo[3,4-c] pyrazol-6(1H)-one (Figure 1), could respectively target these two different pathways with promising selectivity. This hypothesis was supported by a series of biological assays and computational studies.

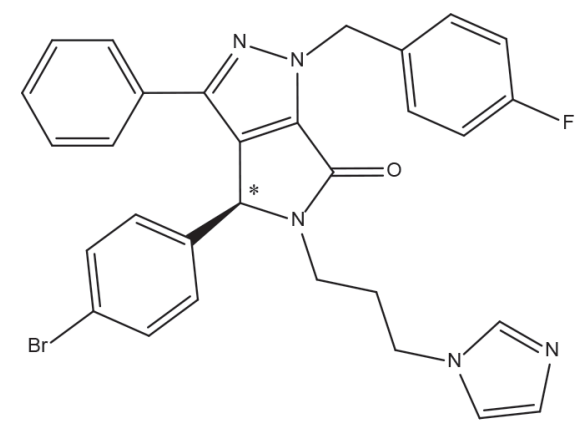

S-5s

\section{RESULTS}

To quantify the level of p53-MDM2 inhibition by the enantiomers of compound $5 \mathrm{~s}$, Western blot analysis was carried out to analyze p53 and MDM2 protein levels in A549 cells. Interestingly, compound R-5s increased the p53 protein level in a dose-dependent manner and inhibited MDM2 expression after 4h treatment (Figure 2), while no significant change was observed in compound $\mathrm{S}-5 \mathrm{~s}$ treated cells. In MCF-7 cells, similar results were observed for $\mathrm{p} 53$, while no significant change was observed for MDMX, a homolog oncoprotein of MDM2 (Figure 3). These findings were consistent with the biochemical assay of $\operatorname{MDMX}\left(\mathrm{K}_{\mathrm{i}}>100 \mu \mathrm{M}\right.$, data not shown).

To determine differences in their bioactivity on the $\mathrm{NF}-\kappa \mathrm{B}$ pathway, the relative cytoplasmic levels of $\mathrm{I} \kappa \mathrm{B} \alpha$ and IKKs from the same cell lysates were examined by Western blot assay (Figure 2). Contrary to the p53 pathway, both enantiomers inhibited phosphorylated I $\kappa \mathrm{B} \alpha$. Compound S-5s exhibited both higher activity and concentration-dependency as compared with R-5s. For $\mathrm{I} \kappa \mathrm{B}$ kinases, incubation of the cells with compound $\mathrm{S}-5 \mathrm{~s}$ only for $4 \mathrm{~h}$ markedly activated the phosphorylation of IKK $\beta$ only or both IKK $\beta$ and IKK $\alpha$ and phosphorylated IKK $\gamma$ in a dose-dependent manner, with no obvious changes in total IKK proteins. Similar behaviour for I $\mathrm{B} \alpha$ with no differences was observed when treated with the other enantiomer, indicating that the compound S-5s of pyrrolo[3,4-c]pyrazole compound suppressed NF- $\mathrm{B}$ activation through inhibition of $\mathrm{I} \kappa \mathrm{B} \alpha$ phosphorylation mediated by the IKKs phosphorylation. The similar but much weaker effect of the other R-5s was probably induced by the p53 pathway rather than by direct inhibition of the NF- $\mathrm{B}$ pathway.

The in vitro antiproliferative activity shown in Table 1 demonstrated that the two enantiomers had a distinct synergistic effect against various cell types.

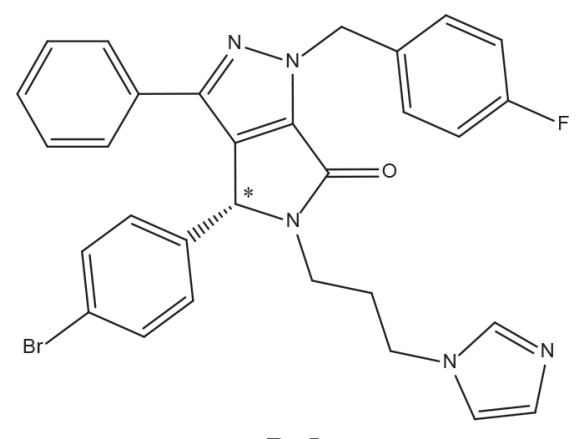

$\mathrm{R}-5 \mathrm{~s}$

Figure 1: Chemical structures of enantiomeric compound R-5s and S-5s. The asterisk indicates the location of the sole chiral center. 


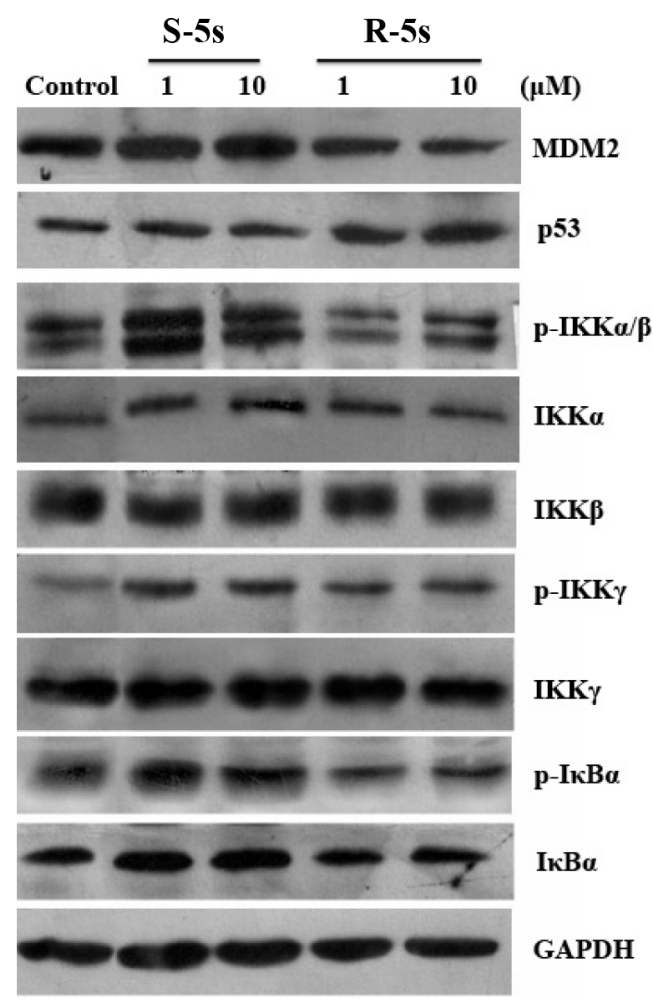

Figure 2: Cellular activity of the two enantiomers on the p53 and NF-кB pathways as detected by Western blotting assay (A549 cells, $4 \mathrm{~h}$ treatment).

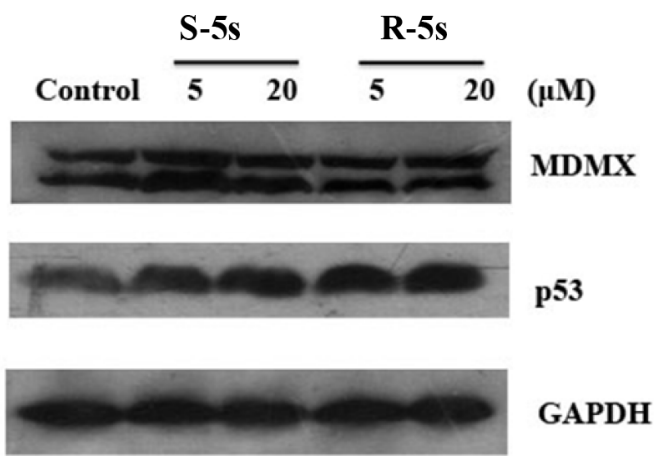

Figure 3: Cellular activity of the two enantiomers on p53 and MDMX as detected by Western blotting assay (MCF-7 cells, MDMX overexpression, $4 \mathrm{~h}$ treatment).

Table 1: In vitro antiproliferative data of compound $5 \mathrm{~s}$

\begin{tabular}{|l|l|l|l|l|}
\hline \multirow{2}{*}{ Compound } & \multicolumn{4}{c}{ MTT-IC $_{\mathbf{5 0}}(\boldsymbol{\mu M})$} \\
\cline { 2 - 5 } & $\mathbf{A 5 4 9}$ & H1299 & U2 OS & \multicolumn{2}{c}{ Saos-2 } \\
\hline Racemic-5s & $5.82 \pm 0.04$ & $2.39 \pm 0.19$ & $2.09 \pm 0.02$ & $5.08 \pm 0.55$ \\
\hline S-5s & $27.4 \pm 1.23$ & $4.02 \pm 0.23$ & $9.2 \pm 1.42$ & $13.5 \pm 1.04$ \\
\hline R-5s & $21.2 \pm 1.52$ & $5.07 \pm 0.14$ & $4.0 \pm 0.30$ & $4.2 \pm 0.16$ \\
\hline Nutlin-3 & $20.79 \pm 1.43$ & $16.34 \pm 5.59$ & $12.76 \pm 2.42$ & $4.14 \pm 0.10$ \\
\hline
\end{tabular}

All the experiments were duplicated. Data are presented as the mean $\pm \mathrm{SD}$. 
The $\mathrm{IC}_{50}$ values of the two enantiomers were 27.4 and $21.2 \mu \mathrm{M}$ against A549 cells, respectively, while the racemic exhibited a non-additive synergistic enhancement of $5.82 \mu \mathrm{M}$. The similar effect was observed in both H1299 and U2 OS cells, but not obviously in Saos-2 cells. Western blot study showed that the S-5s could inhibit NF- $\kappa \mathrm{B}$ activation only, while the R-5s could inhibit both p53-MDM2 interaction and induce the inhibition of $\mathrm{NF}-\kappa \mathrm{B}$ activation with the in vitro antiproliferative result indicating poor selectivity over cancer cell lines (H1299 and Saos-2) with deleted p53.

Considering that both the p53 and NF- $\mathrm{BB}$ were involved in the control of apoptosis $[1,22]$, the effect of the two enantiomers on the induction of A549 cell apoptosis was evaluated by fluorescence-activated cell sorting (Figure 4). After $47 \mathrm{~h}$ treatment at the concentration of $10 \mu \mathrm{M}$, the percentage of apoptotic cells for the two enantiomers was about $4.18 \%$ and $2.92 \%$, respectively, while no significant apoptotic effect was demonstrated in the control group.

To investigate the in vivo effect of the two enantiomers on tumor growtha, an A549 xenograft mouse model was prepared to evaluate compound $5 \mathrm{~s}$ racemic at the dose of $200 \mathrm{mg} / \mathrm{kg}$, while the dose of $100 \mathrm{mg} / \mathrm{kg}$ was used for the two single enantiomers. As depicted in Figure 5, intragastrical (i.g.) administration of these compounds for 18 days significantly inhibited tumor growth $(p<0.05)$. The tumor volume was inhibited by 40.53\% (racemic), 32.35\% (S-5s) and 24.11\% (R-5s), respectively. The two enantiomers were found to be well tolerated during the test and showed no significant loss of body weight as compared with the reference drug doxorubicin (DOX) (Figure 5). The above results also indicate that the two enantiomers had some synergistic effect both in vitro and in vivo.

Docking studies were performed to examine the potential mode of binding for $\mathrm{R}-5 \mathrm{~s}$ and $\mathrm{S}-5 \mathrm{~s}$ in MDM2 (Figure 6). The result showed both enantiomers bind similarly to the crystallographically observed benzodiazepine, indicating that insertion of its aromatic substituents into the three primary sub-pockets of MDM2 was crucial for molecular recognition. The interactions involved were the two phenyl rings with the two hydrophobic I61 and V93 sub-pockets and the diazole ring binding to the H96 sub-pocket via $\pi-\pi$ interaction. The primary scaffold of the pyrrolo[3,4-c]pyrazole group in S-5s occupied the same L54 and G58 region as benzodiazepine with a single hydrogen bond formed between its lactam oxygen atom and L54. This binding conformation also allowed the benzyl group of R-5s to bind outside the binding hotspot region of MDM2 and formed an additional $\pi-\pi$ interaction with F55 which was neither possible in benzodiazepine nor S-5s. The overall docking score for R-5s and S-5s was -7.6 and -6.7 , respectively $v s .-7.6$ of benzodiazepine which could corroborate our observed higher bioactivity of R-5s over $\mathrm{S}-5 \mathrm{~s}$ against the $\mathrm{p} 53-\mathrm{MDM} 2$ pathway.

For IKK $\beta$, the original solved $\mathrm{X}$-ray structure consists of XNM (4-((4-(4-(chlorophenyl) pyrimidin-2-yl) amino) phenyl) (4-(2-hydroxyethyl) piperazin-1-yl) methanone) that binds to a series of connected hydrophobic pockets consisting of L21, V29, A42, I65, Y98, and G102 with hydrogen bonding to K106 (Figure S2). The observed binding mode for both R-5s and $\mathrm{S}-5 \mathrm{~s}$ showed binding poses that recapitulate the interactions of XNM by binding to the same sites with its primary scaffold while extending its halo substituted phenyl ring into the V29 and I26 sub-pocket, forming a salt bridge between its negatively charged diazole group with $\mathrm{K} 105$. The difference in the mode of binding between S-5s and R-5s was the placement of its primary scaffold and the subsequent placement of all four of its aromatic rings into various sub-pockets of IKK $\beta$. For $\mathrm{S}-5 \mathrm{~s}$, the placement of its core scaffold near E149 resulted in a favourable nonbond interaction of nearly $-5 \mathrm{kcal} / \mathrm{mol}$ which was not found in XNM and R-5s. The overall docking score for R-5s and
A

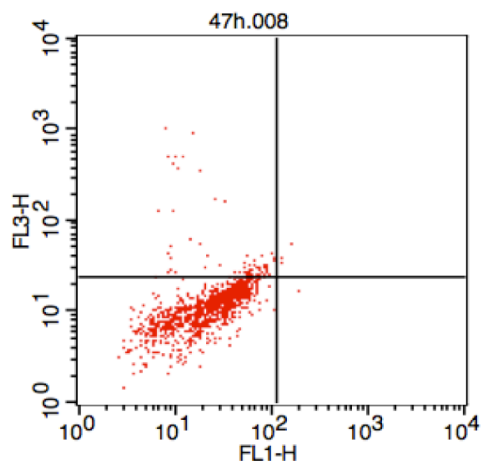

B

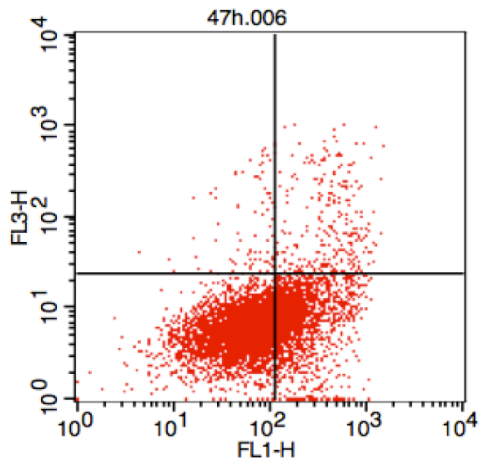

C

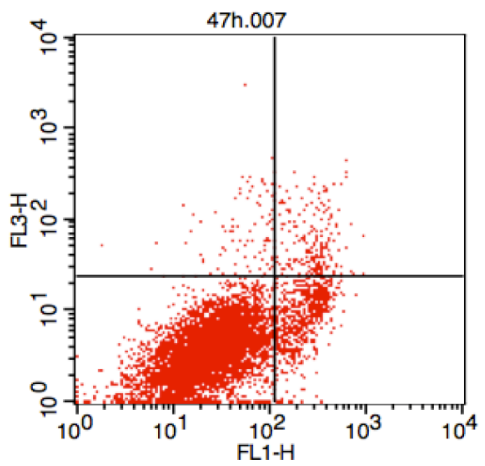

Figure 4: Two enantiomers-induced cell apoptosis. (A) A549 cells were treated with DMSO as control; (B) $10 \mu \mathrm{M}$ of compound S-5s for $47 \mathrm{~h}$; (C) $10 \mu \mathrm{M}$ of compound R-5s for $47 \mathrm{~h}$. 

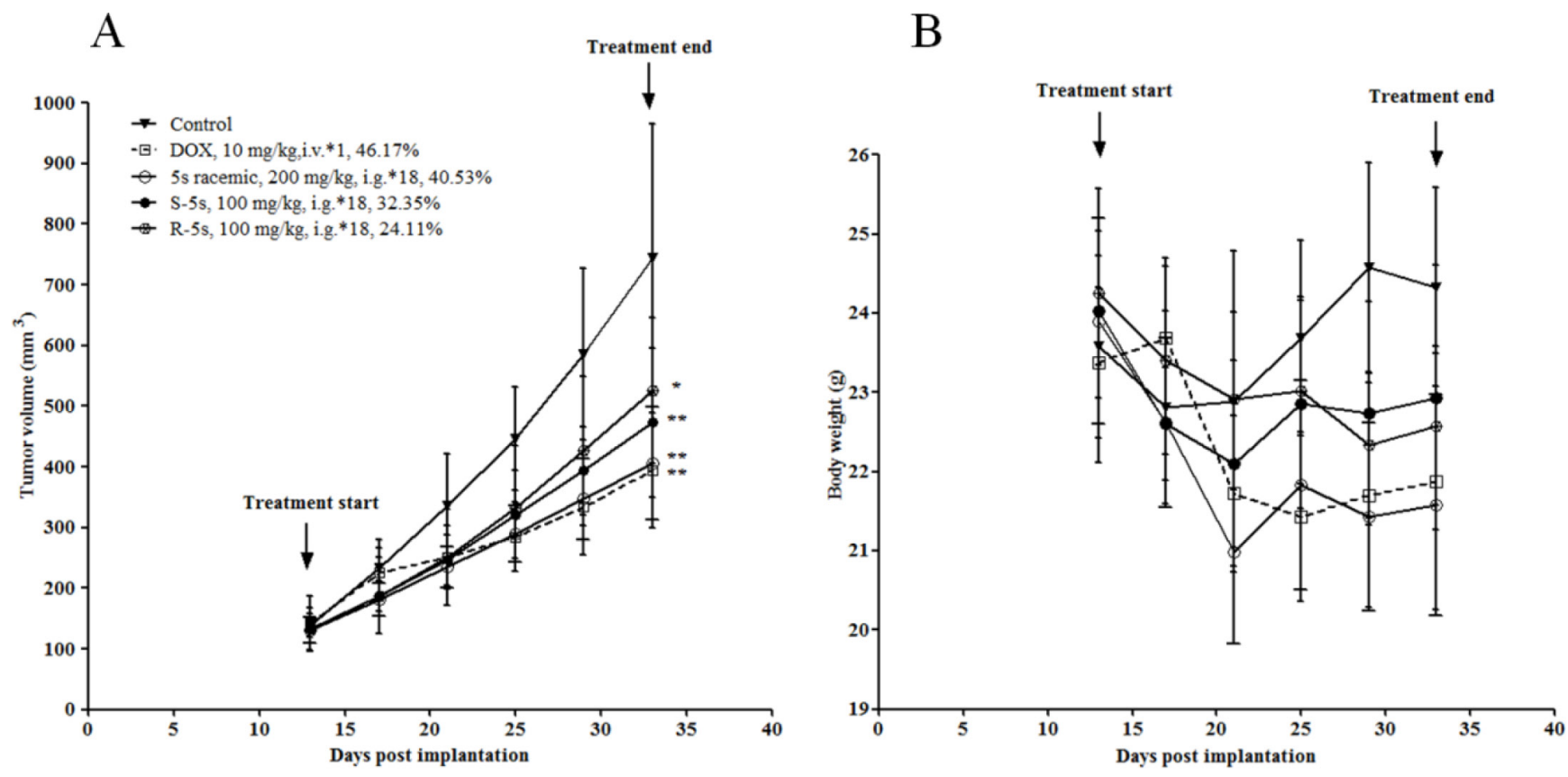

Figure 5: Antitumor activity of compound $5 \mathrm{~s}(200 \mathrm{mg} / \mathrm{kg}$, administered intragastrically, 18 days), and compounds S-5s and R-5s (100 mg/kg, administered intragastrically, 18 days) against A549 xenografts in nude mice. (A) tumor volume; (B) animal body weight. Data are presented as the mean $\pm \mathrm{SEM} ; \mathrm{n}=6$ nude mice per group: $\left.\left(^{*}\right) p<0.05,{ }^{* *}\right) p<0.01$, versus control group $(\mathrm{n}=10$ nude mice), determined with Student's $\mathrm{t}$ test.

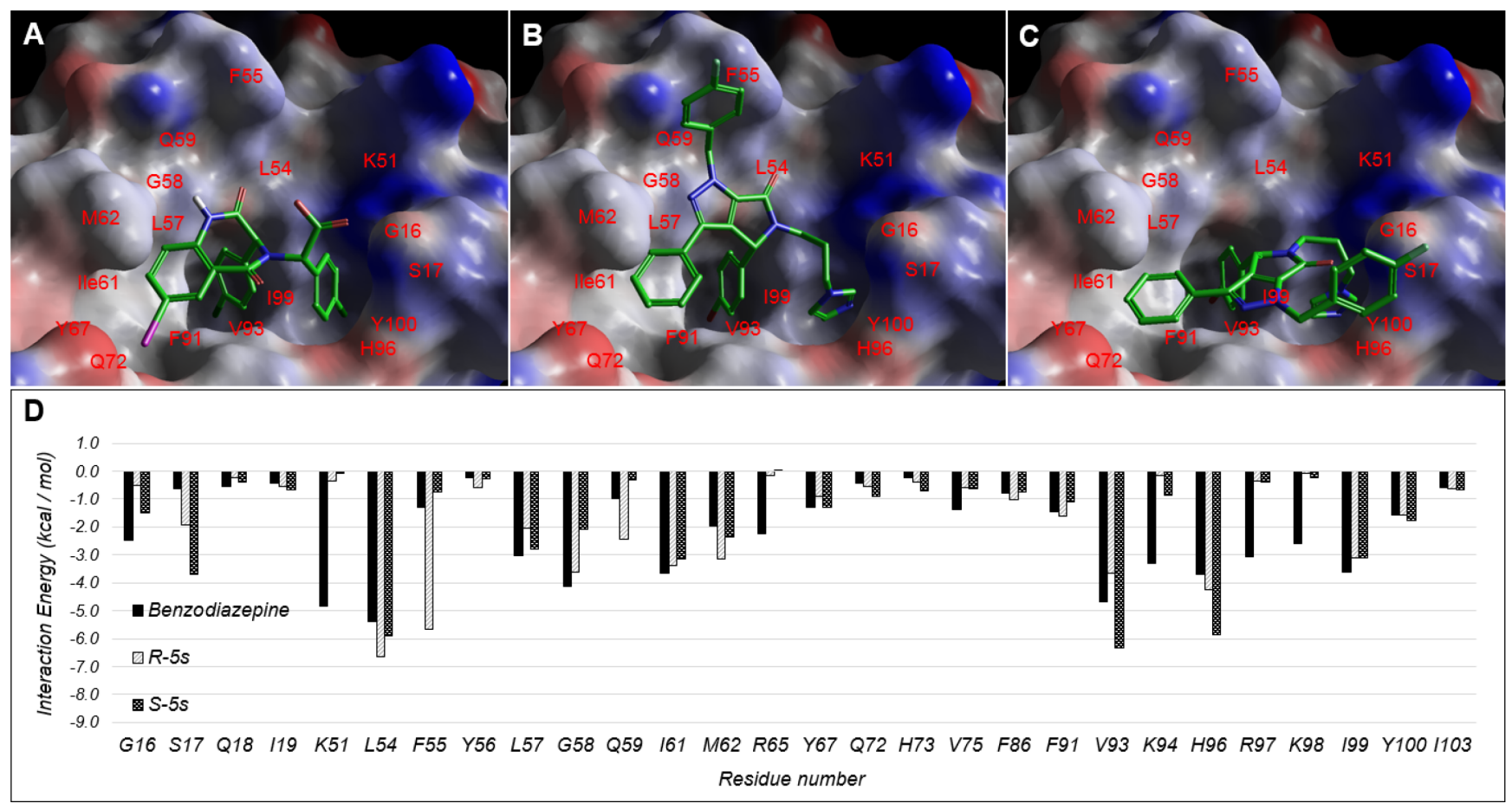

Figure 6: Observed Binding mode of (A) benzodiazepine and docking poses of (B) R-5s and (C) S-5s within the p53-binding site of MDM2. (D) Per residue interaction energy diagram for benzodiazepine (Blue), R-5s (orange) and S-5s (green) within $12 \AA$ of MDM2 ligand binding site. Only interactions greater than absolute $1 \mathrm{kcal} / \mathrm{mol}$ are shown. 
S-5s was -4.1 and -5.4 , respectively $v s$. -7.6 of XNM. The observed docking score was relatively low that suggests both enantiomers could be a relatively weak inhibitor as compared to XNM with $\mathrm{S}-5 \mathrm{~s}$ as the more potent of the two for potentiating the synergistic bioactivities of compound $5 \mathrm{~s}$.

Molecular dynamics (MD) simulations were carried out to further establish the dynamical nature of the essential interactions involved in molecular recognition (Figure 8). The 100ns simulations for the original X-ray structures of MDM2 with benzodiazepine exhibited an average $\mathrm{C}_{\alpha}$ RMSD of $1.0 \AA$ with a standard deviation with $0.1 \AA$, indicating the existence of a tightly bound complex that did not allow significant conformational changes after complexation. For IKK $\beta$ with $\mathrm{XNM}$, the average $\mathrm{C}_{\alpha} \mathrm{RMSD}$ for the ligand binding IKK $\beta$ KD domain was $2.9 \AA$ (Figure S3) with a standard deviation with $0.5 \AA$, indicating a more conformation flexible target. For the two enantiomeric complexes, the simulations showed that the predicted modes of binding were maintained with a relatively slight increase in average $\mathrm{C}_{\alpha} \mathrm{RMSD}$ for MDM2 with R-5s and a decrease in average $\mathrm{C}_{\alpha} \mathrm{RMSD}$ for IKK $\beta$ with $\mathrm{S}-5 \mathrm{~s}$ at $1.5 \AA$ and $2.4 \AA$, respectively. The ability to lower the conformational flexibility of IKK $\beta$ KD domain suggested the potentiality of $\mathrm{S}-5 \mathrm{~s}$ as an allosteric protein-protein interaction inhibitor (PPIi) for modulating IKK $\beta$ 's affinity for the NF- $\kappa \mathrm{B}$ complex.

Finally, the average nearest interatomic distances between the inhibitors and the essential residues with the ligand binding site identified from the docking study are shown in Table 2. For MDM2, all but two of the distances between MDM2 and either benzodiazepine or $\mathrm{R}-5 \mathrm{~s}$ were conserved with less than $1.0 \AA$ differences. $\mathrm{R}-5 \mathrm{~s}$ with an additional methylene spacer between its core scaffold and the diazole group allowed it to penetrate deeper into the His96 sub-pocket as compared with benzodiazepine. Also as shown in the docking study and the per energy interaction diagram, R-5s could extend its benzyl group to Phe55 to form an additional $\pi-\pi$ interaction with an average nearest interatomic distance of $4.6 \AA$. For IKK $\beta$, similar conserved distances were observed including K106 with the exception of E149. The placement of S-5s core scaffold near E149 at the average nearest interatomic distance of $3.4 \AA$ explained why the observed favourable non-bond interaction of $-5 \mathrm{kcal} / \mathrm{mol}$ was distinctively unique from R-5s and XNM (Figure S4).
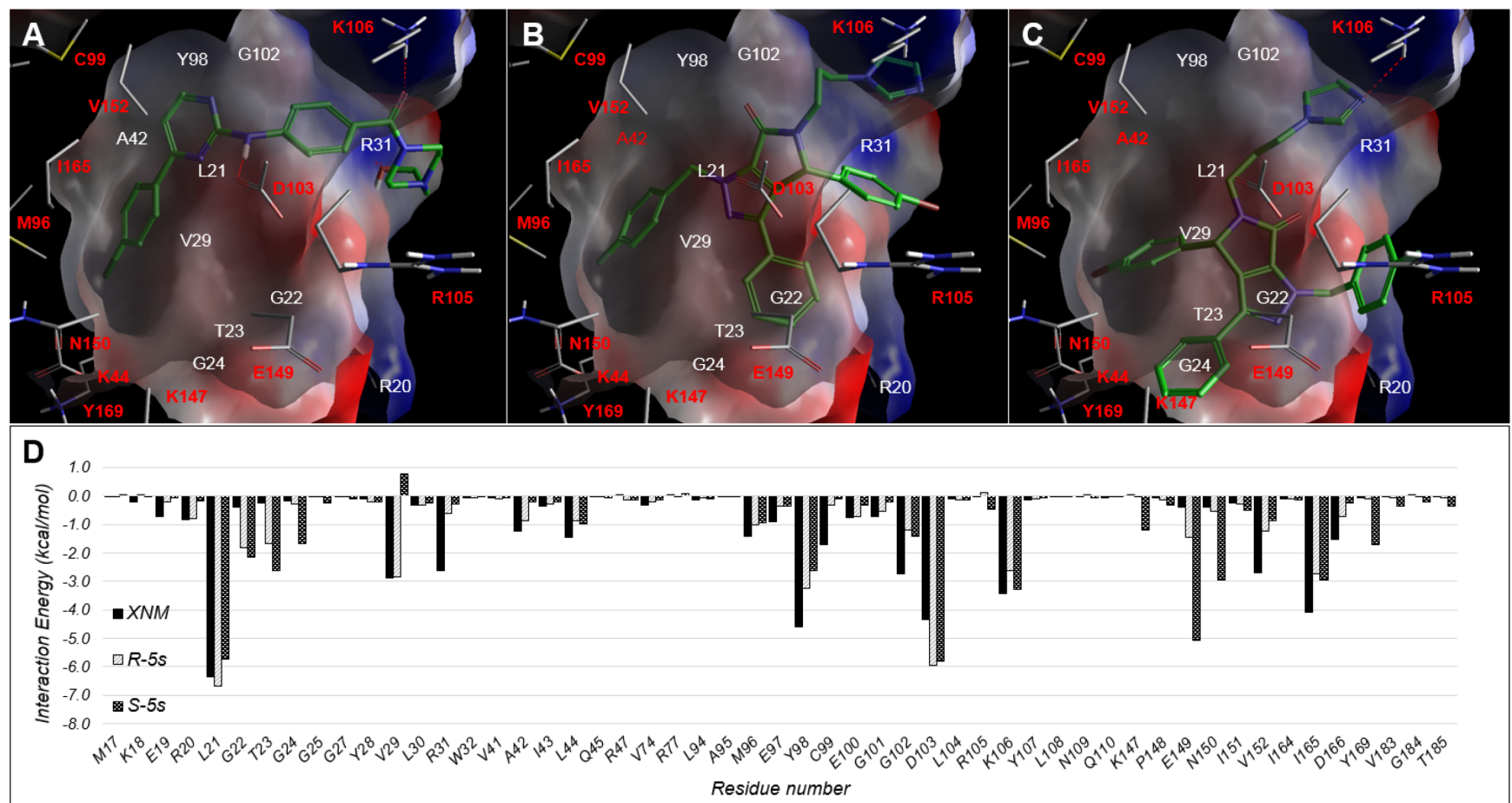

Figure 7: Observed Binding mode of (A) XNM and docking poses of (B) R-5s and (C) S-5s within the ligand binding IKK $\beta$ KD domain. (D) Per residue interaction energy diagram for XNM (Blue), R-5s (orange) and S-5s (green) within $12 \AA \AA$ of MDM2 ligand binding site. Only interactions greater than absolute $1 \mathrm{kcal} / \mathrm{mol}$ are shown. 

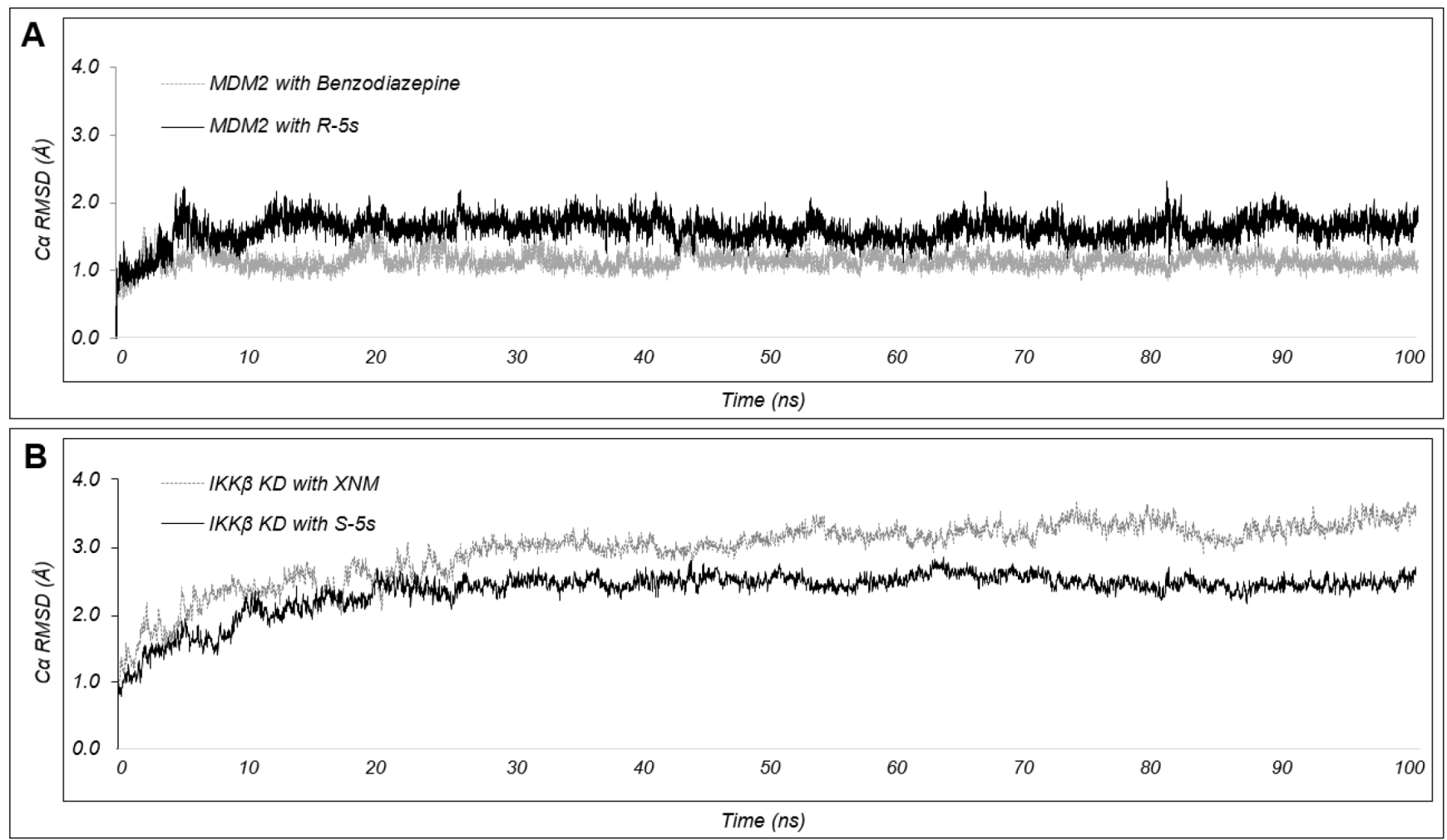

Figure 8: Plot of $C_{\alpha}$ RMSD for the inhibitor bound (A) MDM2 and (B) IKK $\beta$ complexes over the course of 100ns MD simulation. Simulations with the enantiomeric inhibitors (R-5s and S-5s) are shown in black while the structural solved inhibitors (benzodiazepine and XNM) are shown in grey.

Table 2: Average nearest interatomic distances between inhibitors and binding site residues

\begin{tabular}{|c|c|c|c|c|c|}
\hline \multirow{2}{*}{ Residues } & \multicolumn{3}{|c|}{ MDM2 } & \multicolumn{2}{|c|}{ IKK $\beta$} \\
\hline & benzodiazepine & R-5s & Residues & XNM & S-5s \\
\hline Leu54 & 4.2 & 4.5 & Leu21 & 4.2 & 3.8 \\
\hline Phe55 & 6.3 & 4.6 & Met96 & 5.3 & 4.6 \\
\hline Gly58 & 4.2 & 4.3 & Tyr98 & 4.7 & 4.9 \\
\hline Gln59 & 5.5 & 4.4 & Asp103 & 4.5 & 3.8 \\
\hline Ile61 & 3.8 & 4.0 & Lys106 & 4.3 & 4.0 \\
\hline Val93 & 4.0 & 3.8 & Glu149 & 7.6 & 3.4 \\
\hline His96 & 5.5 & 4.6 & Ile165 & 3.6 & 4.0 \\
\hline Ile99 & 3.8 & 3.8 & & & \\
\hline
\end{tabular}

The essential residues within the ligand binding site were identified from the docking study. The distances are averaged over the course of the simulation and are shown in $\AA$.

\section{DISCUSSION}

In the present study, we reported a first-in-class enantiomeric inhibitor with dual efficacy for cancer therapy. Our previous report demonstrated the chiral center played a crucial role in the MDM2 binding activity [21].
For the two enantiomers of compound $5 \mathrm{~s}$ obtained by chiral separation, we found only R-5s could inhibit p53MDM2 interaction and release p53 to suppress tumor proliferation. Molecular modelling further corroborates with our biochemical studies. Like most p53-MDM2 inhibitors, R-5s could mimic the three residues of the $\mathrm{p} 53$ 
and insert into three sub-pockets of MDM2 with a much higher docking Gscore of -7.652 (Figure 6B). However, S-5s interacted with MDM2 by only filling its Phe19 and Trp23 sub-pockets with its two aromatic substituents (Figure 6C).

Interestingly, the inactive p53-MDM2 inhibitor $\mathrm{S}-5 \mathrm{~s}$ demonstrated promising in vitro antiproliferative activity as compared with active enantiomer R-5s. On the other hand, racemic compound $5 \mathrm{~s}$ was illustrated to efficiently suppress NF- $\kappa \mathrm{B}$ activation by inhibiting NF- $\kappa \mathrm{B}$ protein translocation to the nucleus [21]. In this study, we demonstrated that $\mathrm{S}-5$ s could inhibit I $\mathrm{B} \mathrm{B} \alpha$ phosphorylation and markedly activate the phosphorylation of IKK $\beta$ only or both IKK $\beta$ and IKK $\alpha$ and phosphorylated IKK $\gamma$ in a dose-dependent manner only for $4 \mathrm{~h}$. Therefore, S-5s could inhibit NF- $\kappa \mathrm{B}$ signalling via promoting the IKK phosphorylation and preventing the phosphorylation of $\mathrm{I} \kappa \mathrm{B}$ proteins. In addition, most $\mathrm{NF}-\kappa \mathrm{B}$ repressors have been found to be p53 activators and vice versa $[11,12]$. The Western blotting resultshowed that R-5s could inhibit the p53-MDM2 binding in the cells and subsequently induce the NF- $\kappa \mathrm{B}$ inhibition (Figure 2). Computational study predicted that the S-5s had a better docking Gscore $(-5.441)$ with IKK $\beta$ protein than $\mathrm{R}-5 \mathrm{~s}$ (Gscore = -4.143) (Figure 7).

It was hypothesized that drug combination acting more than one target could enhance normal tumor sensitivity and increase therapeutic indices. However, this hypothesis is being challenged clinically because it is no easier to achieve a tolerable drug level. It was found in this study that two enantiomers of one compound could lower the risk of drug combination and targets two different pathways simultaneously. It is also encouraging to see that the two enantiomers had a synergistic effect in three cell lines except Saos-2. For the in vivo efficacy, the enantiomers reduced the tumor volume by $32.35 \%$ for S-5s and $24.11 \%$ for R-5s vs. $40.53 \%$ for the racemic compound $5 \mathrm{~s}(p<0.01)$, which is not significantly lower than $46.17 \%$ for DOX $(p<0.01)$. The above result indicated an apparent synergistic in vivo effect.

In conclusion, we have characterized the mechanism of action of the two enantiomers of pyrrolo[3,4-c] pyrazole compound $5 \mathrm{~s}$. R-5s was found to be potent toward MDM2 and might induce NF- $\kappa$ B inhibition. $\mathrm{S}-5 \mathrm{~s}$ could selectively suppress $\mathrm{NF}-\kappa \mathrm{B}$ activation by inhibiting $\mathrm{I} \kappa \mathrm{B} \alpha$ phosphorylation and the cytoplasmic level elevation of phosphorylated IKKs. The enantiomers had a synergistic effect both in vitro and in vivo. The results of computational analysis further corroborate with the above biological study. The present study has further demonstrated that MD simulations are a powerful tool to predict the atomistic behaviour of tightly binding inhibitors. The obtained interatomic distance measurements indicate that the final binding poses of the two enantiomers are very stable as compare with initial molecular docking model. Further structural optimization to identify the inhibitors of these two pathways with much higher selectivity is ongoing in our laboratory.

\section{METHODS}

\section{In vitro antiproliferative assay}

The cellular growth inhibitory activity was determined using two human osteosarcoma cell lines [U-2 OS and Saos-2] and two human lung cancer cell lines [A549 and NCI-H1299]. An amount of 5-6 $\times 10^{4}$ cells per well was transferred to 96 -well plates. After culturing for $24 \mathrm{~h}$, the test compounds were added to triplicate wells at serial diluted concentrations and $0.1 \%$ DMSO for control. After $72 \mathrm{~h}$ of incubation, $20 \mu \mathrm{L}$ of MTT (3-[4,5-dimethylthiazol-2-yl]-2, 5 -diphenyltetrazolium bromide) solution $(5 \mathrm{mg} / \mathrm{mL})$ was added to each well, and after the sample was shaken for $1 \mathrm{~min}$, the plate was incubated further for $4 \mathrm{~h}$ at $37^{\circ} \mathrm{C}$. The compounds were dissolved in $100 \mu \mathrm{L}$ of DMSO. The absorbance (OD) was quantitated with the microplates using Biotek Synergy $\mathrm{H} 2$ at $570 \mathrm{~nm}$. Wells containing no drugs were used as blanks. The concentration of the compounds that inhibited cell growth by $50 \%\left(\mathrm{IC}_{50}\right)$ was calculated. Nutlin-3 was used as a reference compound.

\section{Western blotting assay}

A549 or MCF-7 cancer cells with wild-type p53 were grown in the recommended medium supplemented with 10\% FBS (Invitrogen) in a humidified environment with $5 \% \quad \mathrm{CO}_{2}$. After $4 \mathrm{~h}$ treatment with various concentrations of the compounds, cells were lysed and the protein extract was denatured and run on 5\% Trisglycine polyacrylamide gels (Invitrogen). Gels were electroblotted onto nitrocellulose membranes, and Western detection was carried out using 5\% milk buffer ( $5 \%$ nonfat dry milk in TBS $/ 0.1 \%$ Tween-20) throughout. Proteins were detected by ECL chemiluminescence reagents (Pierce, \#32209) using antibodies specific for human p53 (Calbiochem, \#OP43T), MDM2 (Millipore, \#07-575), MDMX (abcam, \#ab16058), NF-кB p65 (D14E12) Rabbit mAb (CST, \#8242S), NF- $\mathrm{BB}$ (p105/p50) (NFKB1) antibody (Epitomic, \#1559-1), IKK $\alpha$ (abcam, \#1615-1), IKK $\beta$ (IKBKB) antibody (abcam, \#3902-1), IKK $\gamma$ (DA10-12) Mouse mAb (CST, \#2695S), Phospho-IKK $\alpha / \beta$ (Ser176/180) (16A6) Rabbit mAb (CST, \#2697S), Phospho-IKK $\gamma$

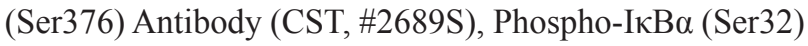
(14D4) Rabbit mAb (CST, \#2859S), IкBa (44D4) Rabbit mAb (CST, \#4812S), GAPDH antibody (multiscience, \#mab5465), and Histone 3 (D1H2) $\mathrm{XP}^{\circledR}$ Rabbit mAb (CST, \#4499P). 


\section{Apoptosis analysis by flow cytometry}

A549 cells $\left(5 \times 10^{5}\right.$ cells $\left./ \mathrm{mL}\right)$ were seeded in six-well plates and treated with the compounds at the concentration of $10 \mu \mathrm{M}$ for $47 \mathrm{~h}$. The cells were then harvested by trypsinization and washed twice with cold PBS. After centrifugation and removal of the supernatants, cells were resuspended in $400 \mu \mathrm{L} 1 \mathrm{X}$ binding buffer, which was then added to $5 \mu \mathrm{L}$ annexin V-FITC and incubated at room temperature for $15 \mathrm{~min}$. After addition of $10 \mu \mathrm{L}$ of PI, the cells were incubated at room temperature for another $15 \mathrm{~min}$ in the dark. The stained cells were analyzed with a flow cytometer (BD Accuri C6).

\section{In vivo antitumor study}

The in vivo antitumor activity of $5 \mathrm{~s}$ and its enantiomers was evaluated using DOX as the reference drug. BALB/C nude male mice (Certificate SCXK20030003, weighing 18-20 g) were obtained from Shanghai Experimental Animal Center of Chinese Academy of Sciences. A549 cancer cell suspension was implanted subcutaneously into the right axilla region of the mice. Treatment was initiated when the implanted tumor grew to a volume of about $120 \mathrm{~mm}^{3}$ in after 13 days. The animals were randomized into appropriate groups (6 animals per treatment group and 10 animals for the control group) and administered by gavage once daily for 18 consecutive days from day 13 after implantation of the cells. Tumor volumes (TV) were monitored by caliper measurement of the length and width and then calculated using the formula: TV $=1 / 2 \times$ $\mathrm{a} \times \mathrm{b}^{2}$, where $\mathrm{a}$ is the tumor length and $\mathrm{b}$ is the width. Tumor volumes and bodyweights were monitored every 4 days throughout the course of treatment. Mice were sacrificed on day 33 after cell implantation, and tumors were removed and recorded for analysis. Tumor volume inhibition was determined by the formula of $1-\mathrm{RTV}_{\text {tumor }} / \mathrm{RTV}_{\text {control }} \times 100 \%$, where RTV is the relative tumor volume to that of day 13. DOX (10 mg/kg, Pfizer Italia S.r.l) was administered intravenously once on day 13 after implantation.

\section{Molecular docking}

The standard protocol for the docking study used for distinguishing the different potential mode of binding among enantiomers has been described in detailed elsewhere [23]. Briefly, All docking studies were carried out using the Schrodinger modeling suite package [24]. The crystallographic structures of the targeted MDM2 (PDB code: 1T4E) [4] and IKK $\beta$ (PDB code: 3RZF) [25] with bound inhibitors were used as the starting point for examining the potential mode of binding for the two enantiomers of compound 5s. All crystallographic waters and ions were removed prior to the addition of the missing hydrogen atoms according to the ionizable states at physiological $\mathrm{pH}$. The prepared protein structures were energy minimized using OPLS-AA 2005 force field [26] to optimize all hydrogen-bonding networks. Both enantiomers of compound $5 \mathrm{~s}$ were built and docked into the prepared protein structure without restraints using the Standard Precision protocol of Schrodinger's Glide v5.6. To identify the key amino residues involved in the molecular recognition that distinguish between the two enantiomers, the non-bond per residue interaction energies between each docked ligand to residues within $12 \AA$ of the targeted binding site were evaluated with a constant dielectric constant of 4 .

\section{Molecular dynamics simulation}

MD simulations were carried out for MDM2 and IKK $\beta$ in complex with the structurally solved inhibitors and with the more potent of the two enantiomers. Each system was solvated in a cubic box with explicit TIP3P water [27] and counter ions consisting of a $10 \AA$ solvent buffer region from the edge of the complex. The long range electrostatic interactions were evaluated by the Particle-Mesh Ewald method under the periodic boundary condition. $100 \mathrm{~ns}$ simulation was carried out for each docked model using DESMOND [28] with OPLS-AA 2005 force field under the isobaric isothermal (NPT) condition at $300 \mathrm{~K}$ and $1 \mathrm{~atm}$. The stability of the simulation was assessed by monitoring the $\mathrm{C}_{\alpha}$ RMSD with respect to the minimized starting structure. For IKK $\beta$ consisting of KD, ULD and SDD domains, the $\mathrm{C}_{\alpha}$ RMSD was evaluated for the ligand binding KD domain.

\section{AUTHOR CONTRIBUTIONS}

Ideas and experiment design: C. Zhuang, C. Sheng, Y. Y. Sham, Z. Miao, W. Zhang.

Computational development: W. S. Shin, Y. Y. Sham. Chemistry and Biology: C. Zhuang, Y. Wu, J. Li, J. Yao, C. Sheng, Z. Miao.

Analysis and data interpretation: C. Zhuang, C. Sheng, W. S. Shin, Y. Y. Sham, Z. Miao.

Writing and review of the manuscript: All the authors Study supervision: Y. Y. Sham, Z. Miao, W. Zhang.

\section{ACKNOWLEDGEMENTS}

We thank the University of Minnesota Supercomputing Institute for providing the necessary computational resources. Financial support for this research is provided by the National Natural Science Foundation of China (grant \# 81373331, 81222044 and 81373278), the $863 \mathrm{Hi}$-Tech Program of China (grant \#2012AA020302), and the Project of Science and Technology of Shanghai (grant \#14431902300).

\section{Conflict of interests}

The authors declare no financial interest in relation to the work described here. 


\section{REFERENCES}

1. Vogelstein B, Lane D, Levine AJ. Surfing the p53 network. Nature. 2000; 408:307-310.

2. Vousden KH, Lu X. Live or let die: the cell's response to p53. Nature reviews Cancer. 2002; 2:594-604.

3. Vassilev LT, Vu BT, Graves B, Carvajal D, Podlaski F, Filipovic Z, Kong N, Kammlott U, Lukacs C, Klein C, Fotouhi N, Liu EA. In vivo activation of the p53 pathway by small-molecule antagonists of MDM2. Science. 2004; 303:844-848.

4. Grasberger BL, Lu TB, Schubert C, Parks DJ, Carver TE, Koblish HK, Cummings MD, LaFrance LV, Milkiewicz KL, Calvo RR, Maguire D, Lattanze J, Franks CF, Zhao SY, Ramachandren K, Bylebyl GR, et al. Discovery and cocrystal structure of benzodiazepinedione HDM2 antagonists that activate p53 in cells. J Med Chem. 2005; 48:909-912.

5. Ding K, Lu Y, Nikolovska-Coleska Z, Qiu S, Ding YS, Gao W, Stuckey J, Krajewski K, Roller PP, Tomita Y, Parrish DA, Deschamps JR, Wang SM. Structurebased design of potent non-peptide MDM2 inhibitors. J Am Chem Soc. 2005; 127:10130-10131.

6. Rew Y, Sun D, Gonzalez-Lopez De Turiso F, Bartberger MD, Beck HP, Canon J, Chen A, Chow D, Deignan J, Fox BM, Gustin D, Huang X, Jiang M, Jiao X, Jin L, Kayser F, et al. Structure-based design of novel inhibitors of the MDM2-p53 interaction. J Med Chem. 2012; 55:4936-4954.

7. Pahl HL. Activators and target genes of Rel/NF-kappa B transcription factors. Oncogene. 1999; 18:6853-6866.

8. Chen LF, Greene WC. Shaping the nuclear action of NF-kappa B. Nat Rev Mol Cell Bio. 2004; 5:392-401.

9. Perkins ND. Integrating cell-signalling pathways with NF-kappa B and IKK function. Nature reviews Molecular cell biology. 2007; 8:49-62.

10. Garg A, Aggarwal BB. Nuclear transcription factor-kappa B as a target for cancer drug development. Leukemia. 2002; 16:1053-1068.

11. Tergaonkar V, Pando M, Vafa O, Wahl G, Verma I. P53 stabilization is decreased upon NF kappa B activation: A role for NF kappa B in acquisition of resistance to chemotherapy. Cancer Cell. 2002; 1:493-503.

12. Dey A, Tergaonkar V, Lane DP. Double-edged swords as cancer therapeutics: simultaneously targeting p53 and NF-kappa B pathways. Nat Rev Drug Discov. 2008; 7:1031-1040.

13. Price DA, Blagg J, Jones L, Greene N, Wager $\mathrm{T}$. Physicochemical drug properties associated with in vivo toxicological outcomes: a review. Expert opinion on drug metabolism \& toxicology. 2009; 5:921-931.

14. Roth BL. Drugs and valvular heart disease. The New England journal of medicine. 2007; 356:6-9.
15. Campillos M, Kuhn M, Gavin AC, Jensen LJ, Bork P. Drug target identification using side-effect similarity. Science. 2008; 321:263-266.

16. Knight ZA, Lin H, Shokat KM. Targeting the cancer kinome through polypharmacology. Nature reviews Cancer. 2010; 10:130-137.

17. Tang J, Maddali K, Dreis CD, Sham YY, Vince R, Pommier Y, Wang ZQ. N-3 Hydroxylation of Pyrimidine-2,4diones Yields Dual Inhibitors of HIV Reverse Transcriptase and Integrase. Acs Med Chem Lett. 2011; 2:63-67.

18. Chen LQ, Petrelli R, Gao GY, Wilson DJ, McLean GT, Jayaram HN, Sham YY, Pankiewicz KW. Dual inhibitors of inosine monophosphate dehydrogenase and histone deacetylase based on a cinnamic hydroxamic acid core structure. Bioorganic \& Medicinal Chemistry. 2010; 18:5950-5964.

19. Besnard J, Ruda GF, Setola V, Abecassis K, Rodriguiz RM, Huang XP, Norval S, Sassano MF, Shin AI, Webster LA, Simeons FR, Stojanovski L, Prat A, Seidah NG, Constam DB, Bickerton GR, et al. Automated design of ligands to polypharmacological profiles. Nature. 2012; 492:215-220.

20. Zhuang C, Miao Z, Zhu L, Dong G, Guo Z, Wang S, Zhang Y, Wu Y, Yao J, Sheng C, Zhang W. Discovery, synthesis, and biological evaluation of orally active pyrrolidone derivatives as novel inhibitors of p53-MDM2 protein-protein interaction. J Med Chem. 2012; 55:9630-9642.

21. Zhuang C, Miao Z, Wu Y, Guo Z, Li J, Yao J, Xing C, Sheng C, Zhang W. Double-Edged Swords as Cancer Therapeutics: Novel, Orally Active, Small Molecules Simultaneously Inhibit p53-MDM2 Interaction and the NF-kappa B Pathway. J Med Chem. 2014; 57:567-577.

22. Levine AJ. p53, the cellular gatekeeper for growth and division. Cell. 1997; 88:323-331.

23. Raza A, Sham YY, Vince R. Design and synthesis of sulfoximine based inhibitors for HIV-1 protease. Bioorganic \& Medicinal Chemistry Letters. 2008; 18: 5406-5410.

24. Maestro 9.3 G, Prime 3.1, Macromodel 9.9, Desmond 3.1; Schrodinger, LLC: New York, NY, 2012.

25. Xu GZ, Lo YC, Li QB, Napolitano G, Wu XF, Jiang XL, Dreano M, Karin M, Wu H. Crystal structure of inhibitor of kappa B kinase beta. Nature. 2011; 472: 325-U222.

26. Jorgensen WL, Maxwell DS, Tirado-Rives J. Development and testing of the OPLS all-atom force field on conformational energetics and properties of organic liquids. J Am Chem Soc. 1996; 118:11225-11236.

27. Jorgensen WL, Chandrasekhar J, Madura JD, Impey RW, Klein ML. Comparison of simple potential functions for simulating liquid water. J Chem Phys. 1983; 79: 926-935.

28. Research DES. (2008). Desmond Molecular Dynamics System. (New York, NY). 